\title{
AtHDA15 Attenuates COP1 via Transcriptional Quiescence, Direct Binding, and Sub- Compartmentalization During Photomorphogenesis
}

Malona Velasco Alinsug ( $\square$ malinsug@yahoo.com )

Mindanao State University - General Santos https://orcid.org/0000-0001-9279-0705

Custer C. Deocaris

Philippine Nuclear Research Institute

\section{Research Article}

Keywords: COP1, HDA15, histone deacetylases, light signal transduction, photomorphogenesis, protein interaction, Arabidopsis thaliana

Posted Date: March 15th, 2021

DOl: https://doi.org/10.21203/rs.3.rs-249294/v1

License: (c) (1) This work is licensed under a Creative Commons Attribution 4.0 International License.

Read Full License 


\section{Abstract}

Light is an essential environmental cue that determines the overall growth and development of plants. However, the molecular mechanisms underpinning the light signaling network are obscured by the epigenetic machinery where reversible acetylation and deacetylation play crucial roles in modulating light-regulated gene expression. In this paper, we demonstrate that HDA15 represses COP1, the master switch in the light signaling network, by deacetylation, protein interaction, and sub-compartmentalization. hda 15 T-DNA mutant lines exhibited light hyposensitivity with significantly reduced HY5 and PIF3 transcript levels leading to long-hypocotyl phenotypes in the dark while its overexpression exhibited elevated HY5 transcripts and short hypocotyl phenotypes. In vivo and in vitro binding assays further show that HDA15 directly interacts with COP1 inside the nucleus modulating COP1's repressive activities. Crossing hda15-t27 with cop 1-4 mutants resulted in short-hypocotyl and dwarfed phenotypes, reminiscent of cop1-4 mutants suggesting COP1 is epistatic to HDA15. Although light signals the nucleocytoplasmic shuttling of HDA15, the presence of COP1 triggers its nuclear localization. A working model is presented elucidating the concerted interplay between HDA15 and COP1 under light and dark conditions.

\section{Introduction}

Plants adapt extensively to the dynamic changes in their light environment as they are critically dependent on light for photosynthesis, growth, and development. In general, light induces massive changes in a plant's transcriptional activity resulting in distinct expression profiles during seedling development (Jiao et al., 2007; Ma et al., 2013). Approximately 15\% of these transcriptional changes have been attributed to histone modifications, specifically the acetylation in H3K9K27, which coincides with the upregulation of key photomorphogenesis gene expression, such as HY5 and its homolog, HYH (Charron et al., 2009). H3K9ac is one of the most characterized epigenetic marks invariably correlated with transcriptional activation in all the species studied (Ausin et al., 2004; Kurdistani et al., 2004; Schübeler et al., 2004; Benhamed et al., 2008; Guo et al., 2008; Charron et al., 2009; Zhou et al., 2009; Schenke et al., 2014; Liu et al., 2017). However, most of these light-induced pathways remain obscure, while little is understood about histone deacetylases' involvement in regulating these genes.

Studies on histone active marks such as H3K9K27ac have been limited to positive photomorphogenic regulators such as CABs, LHBIB1, LHCA1, HYH and HY5 and its downstream targets CHS, IAA3, RBCS-1a (Benhamed et al., 2006; Guo et al., 2008; Charron et al., 2009). Among the 12 members of the RPD3/HDA1 superfamily in Arabidopsis, HDA19, HDA6, and HDA15 have been ascribed active roles in light-regulated gene expression. Based on studies, HDA19 and HDA6 play a negative regulatory role by repressing some of these light-regulated genes (Benhamed et al., 2006; Tessadori et al., 2009). On the other hand, HDA15 has recently been shown to have a regulatory role in photomorphogenesis. As human Class II HDACs are known to undergo nucleocytoplasmic shuttling, we have demonstrated that HDA15 shuttles to the nucleus under light (Alinsug et al., 2012). Moreover, phytochrome interacting factor 3 (PIF3) associates with HDA15 repressing chlorophyll biosynthesis and photosynthesis in etiolated 
seedlings (Liu et al., 2013). Similarly, PIF1 recruits HDA15 at the promoter sites repressing the transcription of light-responsive genes involved in multiple hormonal signaling pathways and cellular processes in germinating seeds in the dark (Gu et al., 2017). Furthermore, HDA15 forms a complex with nuclear factor homologs (NF-YC) functioning as a transcriptional co-repressor via deacetylation inhibiting hypocotyl elongation in photomorphogenesis (Tang et al., 2017). A recent study describes that long hypocotyl 5 (HY5) protein interacts with HDA15 repressing hypocotyl cell elongation during photomorphogenesis (Zhao et al., 2019).

HY5 and PIFs are key transcription factors playing crucial regulatory roles in photomorphogenesis and skotomorphogenesis, respectively. Along with the light signaling network, both HY5 and PIFs are repressed by COP1, an E3 ubiquitin ligase that targets both photoreceptors HY5 and HYH for ubiquitination and proteasome degradation in the dark (Osterlund et al., 2000). However, the molecular interplay between COP1, HY5, PIFs, and HDA15 remains unclear. It remains obscure as to how or when HDA15 precisely interjects within the light response molecular network. As epigenetic regulation further adds complexity to the light signaling pathway, we attempt to explore the multiple roles HDA15 plays during photomorphogenesis. In this paper, we demonstrate that HDA15 represses COP1, the master switch in the light signaling network, by transcriptional silencing via deacetylation, protein-protein association, and sub-compartmentalization. A working model is presented elucidating the interplay of the multiple actions exerted by HDA15 on COP1 under light and dark conditions.

\section{Materials And Methods}

\section{Plant Material}

Seeds of T-DNA knockout lines hda5-t24 (SALK_030624), hda15-t27(SALK_004027C), and hda18-t (SALK_006938C) in Col-0 background were purchased from ABRC and screened via PCR using specific primers. Homozygous mutant plants with positive T-DNA inserts were grown under long day and assessed for the expression of the corresponding gene compared to wild type via RT- PCR using specifically designed primers. Seeds of homozygous T-DNA knockout lines were then collected and further used for this study. To establish the double mutant line, we crossed the homozygous hda15-t27 mutant with the cop 1-4 mutant. The resultant F2 lines were then finally screened by PCR.

\section{Protoplast PEG Transfection}

Leaves of 3-week old Col-0 wild type, T2 transgenic lines, and mutant lines phyA, phyB, cry $1 /$ cry 2, and cop 1-4 were used for protoplast transient expression assay. Isolation of Arabidopsis mesophyll protoplasts and PEG transfection were done as described previously (Yoo et al., 2007) with some modifications. Mesophyll protoplasts were isolated from 3-week old Col-0 plants and Arabidopsis PSB-D cell lines. Twenty $\mu \mathrm{g}$ of HDA15-YFP/GFP fusion plasmid and VirD2-NLS, a nuclear marker, were cotransfected into protoplasts using polyethylene glycol (PEG) solution $(0.4 \mathrm{~g} / \mathrm{ml}$ PEG 4000, $0.8 \mathrm{M}$ mannitol, $125 \mathrm{mM} \mathrm{CaCl}_{2}$ ). After incubation for 5-15 $\mathrm{min}$ at room temperature, the protoplasts were 
washed and resuspended in W5 solution (154 mM NaCl, $125 \mathrm{mM} \mathrm{CaCl}, 5 \mathrm{mM} \mathrm{KCl}, 2 \mathrm{mM}$ MES at pH5.7), and further incubated under white light for 16-24 $\mathrm{h}$ before imaging using Leica SP5 confocal microscope. For the localization of HDA15 in different light treatments, transfected protoplasts were incubated under white light for $18 \mathrm{~h}$ then transferred to E30LEDL3 growth chambers (Percival Scientific) with far-red, red, and blue light-emitting diode sources for $3 \mathrm{~h}$. Low light intensities used as treatment were measured at $2.77 \mu \mathrm{mol} \mathrm{m} \mathrm{m}^{-2}(\mathrm{FR}), 1.77 \mu \mathrm{mol} \mathrm{m} \mathrm{m}^{-2}(\mathrm{R})$, and $3.84 \mu \mathrm{mol} \mathrm{m} \mathrm{m}^{-2} \mathrm{~s}^{-2}(\mathrm{~B})$.

\section{Light treatments and hypocotyl measurements}

Arabidopsis seeds were surface sterilized, cold treated for $3-4$ days and sown on $1 / 2$ MS plates with $0.1 \%$ sucrose then subjected to different light treatments at $21^{\circ} \mathrm{C}$ for $5-7$ consecutive days. White light intensity was approximately $130 \mu \mathrm{mol} \mathrm{m} \mathrm{m}^{-2} \mathrm{~s}^{-1}$. Plated seeds were incubated in E30LEDL3 colored light chambers (Percival Scientific) with the corresponding low $(\mathrm{L})$ and high $(\mathrm{H})$ wavelength measurements as follows: far-red at $18.065(\mathrm{H})$ and $2.77(\mathrm{~L}) \mu \mathrm{mol} \mathrm{m} \mathrm{m}^{-2} \mathrm{~s}^{-1}$, red light at $22.938(\mathrm{H})$ and $1.77(\mathrm{~L}) \mu \mathrm{mol} \mathrm{m} \mathrm{m}^{-2} \mathrm{~s}^{-1}$, and blue light at $11.095(\mathrm{H})$ and $3.84(\mathrm{~L}) \mu \mathrm{mol} \mathrm{m} \mathrm{m}^{-2} \mathrm{~s}^{-1}$, respectively. On the other hand, dark treated seedlings were wrapped in foil and placed inside a dark growth chamber. Hypocotyl length was measured using UTHSCA Image Tool using 30-50 seedlings for each line and further analyzed for statistical tests.

\section{Total RNA extraction \& qRT-PCR}

Gene expression was initially assessed by semi-quantitative RT-PCR. Total RNA was extracted from plant samples weighing 0.25 to $0.3 \mathrm{~g}$ using TRIZOL reagent (Invitrogen). Oligo(dT) primed reverse transcription of first-strand cDNA synthesis was carried out with $7 \mathrm{mg}$ total RNA using Super ScriptTM III (Invitrogen). Equal volumes of each first-strand reaction were amplified with gene-specific primer pairs. Thermocycling conditions were $95^{\circ} \mathrm{C}$ for 4 mins followed by 30 cycles of $95^{\circ} \mathrm{C}$ for $30 \mathrm{~s}, 55-60^{\circ} \mathrm{C}$ for $30 \mathrm{~s}$, and $74^{\circ} \mathrm{C}$ for 1-2 min. cDNAs obtained from RT were then used as templates to run real-time PCR. The following components were added to a reaction tube: $9 \mu \mathrm{L}$ of iQ SYBR Green Supermix solution (Bio-Rad), $1 \mu \mathrm{L}$ of 5 $\mathrm{mM}$ specific primers, and $8 \mu \mathrm{L}$ of the diluted template. Thermocycling conditions were as follows: $95^{\circ} \mathrm{C}$ for $3 \mathrm{~min}$ followed by 40 cycles of $95^{\circ} \mathrm{C}$ for $30 \mathrm{~s}, 60^{\circ} \mathrm{C}$ for $30 \mathrm{~s}$, and $72^{\circ} \mathrm{C}$ for $20 \mathrm{~s}$, with a melting curve detected at $95^{\circ} \mathrm{C}$ for $1 \mathrm{~min}, 55^{\circ} \mathrm{C}$ for $1 \mathrm{~min}$, and detection of the denature time from $55^{\circ} \mathrm{C}$ to $95^{\circ} \mathrm{C}$. Each sample was quantified in triplicate and normalized using Ubiquitin10 as the internal control. Genespecific primer pairs were designed and are available upon request.

\section{Chromatin Immunoprecipitation (ChIP) assay}

ChIP assay was performed as described (Gendrel et al., 2005). Chromatin extracts were prepared from light treated 4-day old seedlings treated with formaldehyde. The chromatin was sheared to an average length of 500 bp by sonication and immunoprecipitated with anti-acetylated histone H3K9K14 (catalog no. 06-599; Millipore). The DNA cross-linked to immunoprecipitated proteins was analyzed by real-time PCR. Relative enrichments of various regions of COP1 over Col-0 were calculated after normalization to 
Ubiquitin10. Each of the immunoprecipitations was tested and analyzed in triplicates, and each sample was quantified at least three times.

\section{Protein Extraction and Gel-Blot Analysis}

Total protein extraction was conducted as described (Hsieh et al., 2000). Four-day old seedlings of wild type Col-0, all mutant, and overexpression lines were continuously grown under different light treatments and duration. After the treatment, the seedlings were harvested and frozen immediately for protein extraction. One hundred micrograms of total protein were separated on a $12 \%$ SDS-polyacrylamide gel, blotted on immuno-blot PVDF (Bio-Rad), and detected using anti-HY5, anti-COP1, and anti-RPN6 specific antibodies.

\section{Protein-Protein Interaction}

For the bifluorescence complementation (BiFC) assay, HDA15 and COP1 coding sequences were cloned into the $\mathrm{PCR} 8 / \mathrm{GW} / \mathrm{TOPO}$ vector and used for recombination into its corresponding destination vectors, pEG201-N-YFP and pEG202-C-YFP, using LR mix. Kanamycin was used for bacterial selection. Purified plasmids were then analyzed for DNA sequencing for confirmation and further used for protoplast PEG transfection and imaging.

Pull-down assay was carried out as described (Yang et al., 2008) with some modifications. For GST pulldown, GST and GST-HDA15 recombinant proteins were incubated in $30 \mathrm{~mL}$ of GST resin with binding buffer (50 mM Tris-Cl, pH 7.5, $100 \mathrm{mM} \mathrm{NaCl}, 0.25 \%$ Triton X-100, and $35 \mathrm{mM}$ b-mercaptoethanol) for two $\mathrm{h}$ at $4^{\circ} \mathrm{C}$. The binding reaction was washed three times with the binding buffer, and then the COP1-His recombinant protein was added and incubated for an additional two $\mathrm{h}$ at $4^{\circ} \mathrm{C}$. For His pull-down, His and COP1-His recombinant proteins were incubated in $30 \mathrm{~mL}$ of His resin in a phosphate buffer $(10 \mathrm{mM}$ $\mathrm{Na} 2 \mathrm{HPO} 4,10 \mathrm{mM} \mathrm{NaH} 2 \mathrm{PO} 4,500 \mathrm{mM} \mathrm{NaCl}$, and $10 \mathrm{mM}$ imidazole) for two $\mathrm{h}$ at $4^{\circ} \mathrm{C}$, the binding reaction was washed three times with the phosphate buffer, and then GST-HDA15 recombinant protein was added and incubated for an additional two $\mathrm{h}$ at $4^{\circ} \mathrm{C}$. After extensive washing (for at least eight times), the "pulled-down" proteins were eluted by boiling, separated by $10 \%$ SDS-PAGE, and detected by western blotting using anti-His, anti-GST, or anti-COP1 specific antibody.

\section{Bioinformatics analysis}

The protein sequences of HY5 and PIF3 were retrieved from the NCBI's protein sequence repository database (www.ncbi.nlm.nih.gov/). Putative protein acetylation sites were predicted by Bayesian based algorithm for protein lysine acetylation (Li et al., 2009).

\section{Statistical analysis}

Statistical analyses were conducted using SigmaStat 11 software. One-way ANOVA using the Holm-Sidak method was performed for multiple pairwise comparisons. Similarly, Dunnette's method was conducted for multiple comparisons using WT as control. However, Kruskal-Wallis One-way ANOVA on Ranks was 
used to analyze data that failed to pass the Normality test. Significance testing was set at an a of 0.05 or 0.01. Graphs were presented in mean values with error bars set from its standard deviation.

\section{Results}

\section{Class II HDA mutant lines display light hyposensitivity}

T-DNA SALK lines of Class II histone deacetylases were screened, analyzed, and grown under various light conditions where long hypocotyl phenotypes were initially observed. To determine the maximum phenotypic expression of the mutant lines, plants were exposed to varying light fluence rates, light intensities, and durations. Seeds of knockout lines were grown under white light and dark treatment for seven consecutive days. Class II mutant lines were likewise grown under continuous far-red, red, and blue light at high and low light intensities for five consecutive days. Hypocotyl lengths were measured on the 3rd, 5th, and 7th days (Figure 1).

Results indicate that loss-of-function lines of HDA5, HDA15, and HDA18 were generally normal under white light treatment. However, in the absence of light, significantly longer hypocotyls starting on the $3^{\text {rd }}$ day for hda 15-t27 and $4^{\text {th }}$ day for hda5-t24 and hda18-t were observed. The hyper-skotomorphogenic phenotype exhibited by Class II HDA mutants after four days of dark treatment was noticeably pronounced as those displayed by the phy $A$ and phyB mutants. This behavior suggests that HDA15 and HDA18 may act downstream of the phytochrome signaling pathway.

Furthermore, light hyposensitive phenotypes of T-DNA knockout mutants were significantly prominent at low light intensities after 3 to 4 days of continuous light treatment. Under far-red, red, and blue light treatments, four days of constant light treatment not only induced long hypocotyls but also yielded bigger, more developed open cotyledons with longer petioles indicating that Class II histone deacetylases might act as positive photomorphogenic regulators. Hypocotyl length of mutant lines eventually leveled off with the wildtype on the fifth day of light treatment, indicating that these histone deacetylases could be active at the early stages of growth and development.

All in all, among all the Class II HDAs tested, hda15-t27 mutants had the most prominent hyposensitive phenotype in both light and dark conditions. Thus, this study focused on elucidating the function of HDA15 in photomorphogenesis.

\section{HDA15 targets the deacetylation of COP1 promoter}

Like the long-hypocotyl phenotype displayed by the hy 5 mutant, we hypothesize that HDA15 acts as a positive regulator of photomorphogenesis. To determine if HDA15 is indeed involved in photomorphogenesis, expression levels of downstream positive regulators of photomorphogenesis were assessed via RT-PCR and qPCR. As presented in Figure 2, among the key positive regulators, which are known to be targeted by acetylation upon light exposure, include HY5, LHB1B1, LHCA1, RBCS-1a, and 1b, CAB1, and CAB2 (Guo et al., 2008; Charron et al., 2009). However, most of these genes, including HY5, 
were significantly reduced in all the light treatments, which explain the long-hypocotyl phenotype suggesting that a negative regulator catapulted the downregulation of these genes, possibly targeted by HDA15.

To further assess the regulatory role of HDA15, mRNA expression profiles of key regulators of photomorphogenesis in hda 15 mutant and overexpression lines were conducted (Figure 3 ). Compared to white light and far-red light treated plants, dark-treatment has eminently amplified HY5 transcripts levels up to 7-folds in HDA15 overexpression lines. Concomitantly, mRNA levels of COP1 were significantly elevated 3-folds in hda 15 mutants, while its overexpression leads to COP1's extreme downregulation compared to wild type. ChIP assay using anti-H3K9K14ac further reveals that the promoter and start sites of COP1 were highly acetylated in the absence of HDA15 in dark-treated plants. The comparative acetylation profiles suggest that HDA15 targets the deacetylation of COP1 in the dark.

\section{Light signals HDA15 nuclear localization, dark treatment induces cytoplasmic translocation}

Hypocotyls of 4-day old HDA15-GFP transformants were used to determine its localization under varying light conditions (Figure 4). Under white light conditions, HDA15 remained to be prominently nuclear. When grown under far-red light, HDA15-GFP concentrated in the nucleus, which is similar to blue and red light treated lines. Consequently, seedlings grown in the dark exhibited strong signals at the cytoplasmic area, although small quantities were still detected at the nucleus. Similar results were observed when HDA15GFP were transiently expressed in protoplasts under various light treatments.

As mammalian Class II HDAs undergo nucleocytoplasmic shuttling, plant Class II HDAs HDA15, in particular, likewise exhibit similar functional regulatory mechanisms. This was further illustrated by Alinsug et al. (2012) where HDA15 depends on its own NLS and NES signals for nucleocytoplasmic shuttling. As revealed in their study, HDA15-GFP transfected protoplasts exhibited nuclear localization after 18 hours of white light incubation. Further dark treatment for 3 hours elicited partial cytoplasmic translocation of HDA15-GFP. After one hour, re-exposure of the protoplasts to white light led to its complete nuclear import. The observed response demonstrates that light indeed drives the nucleocytoplasmic shuttling of HDA15.

\section{COP1 triggers the nuclear localization of HDA15}

Scanning through the amino acid sequence of HDA15, it contains a type par4 nuclear localization signal (NLS) near the amino end, two overlapping bipartite NLS near the carboxyl end, and a nuclear export signal (NES) near the $C$ terminal half. Our previous study has indicated that these signals navigate the subcellular compartmentalization of HDA15, and that light, in general, drives the nucleocytoplasmic shuttling of HDA15 (Alinsug et al., 2012). Albeit the exact mechanisms controlling its NLS and NES signals upon light exposure remains obscure. Thus, it is speculated that the only way for HDA15 to detect light is through the photoreceptors; their inactivation upon the termination of light may have initiated HDA15's nuclear export. 
To test this hypothesis, HDA15-GFP/YFP was transiently expressed in protoplasts of mutant photoreceptors phyA, phyB, and cry1xcry2. As shown in Figure 5, all these mutant photoreceptor protoplasts displayed nuclear localization of HDA15 suggesting that the nuclear import of HDA15 is not dependent on light quality nor controlled singly by the phytochrome/cryptochrome signaling cascades, but implicates the involvement of all photoreceptors. The downstream target of all four photoreceptors, COP1, appears to have a significant impact on HDA15's nuclear localization, where cop1-4 mutant protoplasts exhibited speckled distribution of HDA15 in the cytoplasm immediately near the vicinity of the nucleus. Overall, our results suggested that the activation of photoreceptors did not trigger HDA15 nuclear localization. Instead, the shuttling observed was likely attributed to the presence of COP1.

\section{HDA15 directly interacts with COP1 inside the nucleus}

The human Class II HDAC6 has been well studied to catalyze non-histone proteins such as a-tubulin, cortactin, and HSP90, as well as, bind to ubiquitinated proteins inhibiting their proteosomal degradation via its zinc finger ubiquitin-binding protein (ZnF-UBP) domain (Hook et al., 2002; Kovacs et al., 2005; Luxton and Gundersen, 2007; Valenzuela-Fernandez et al., 2008). Although the corresponding plant ortholog of HDAC6 is unclear, HDA15 contains a RanBP zinc finger analogous to ZnF-UBP of HDAC6. To determine if HDA15 can associate with non-histone proteins such as COP1, pull-down and BiFC assays were conducted (Figure 6). Direct binding between HDA15 and COP1 was illustrated via pull-down assay using anti-His, anti-GST, and anti-COP1 antibodies where HDA15 and COP1 were fused with GST- and Histags, respectively. This interaction was further confirmed in vivo using BiFC demonstrating their direct binding inside the nucleus both under white light and dark conditions. Although it has been consistently demonstrated that HDA15 is nuclear under all the light treatments, the abrogation of COP1 in cop1-4 mutant protoplasts renders it cytoplasmically speckled.

\section{HDA15 positively regulates photomorphogenesis by repressing COP1}

It has long been hypothesized that COP1 may recruit deacetylases or chromatin remodeling factors that can repress transcription from target promoters (Holm and Deng, 1999). If HDA15 functions as a corepressor of COP1, then the mutant lines of HDA15 should exhibit cop 1-like phenotypes. On the contrary, hda15-t27 lines manifested light hyposensitivity similar to hy 5 mutants with down-regulated levels of HY 5 and other positive regulators of photomorphogenesis. Thus, HDA15 may indirectly regulate HY 5 by repressing COP1, making it a positive regulator of photomorphogenesis.

To establish the genetic relationship between HDA15 and COP1, we generated the double mutant cop14xhda15-t27. As shown in Figure 7, the cross between hda15-t27 and cop1-4 mutants exhibited short hypocotyl phenotypes under white light and dark treatments and dwarfed phenotypes in 3-week old plants all reminiscent of cop1-4 mutants. These responses suggest that COP1 is epistatic to HDA15. Therefore, HDA15 targets the repression of COP1 via direct binding, thereby attenuating its capacity to ubiquitinate HY 5 and its targets.

\section{Ubiquitinated sites of HY5 and COP1 are less stable than HDA15}


Considering that COP1 is an E3 ligase, the binding of HDA15 with COP1 raises pertinent questions as to whether COP1 ubiquitinates HDA15 or HDA15 represses COP1. Aside from the double mutant cross between had15 and cop1 resulting to cop1-like phenotype, bioinformatics analysis using three algorithms, namely: Ub-Pred, BDM-PUB, and UbiSite, were used to predict ubiquitination sites comparing HY5, COP1, and HDA15. Based on the algorithms' consensus, as presented in Table 1, HY5 was found to contain five residues that are actively targeted for ubiquitination, rendering it the most unstable among the three, with instability index of 68.21. COP1 follows HY5 with an instability index of 47.13 , with four consensus residues prone to ubiquitination. On the other hand, it appears that HDA15 is the most stable among the three proteins with an instability index of 37.11 , noting that it only has two residues as prospective sites for ubiquitination. This bioinformatics analysis hence reaffirms our prior notion that HY 5 is targeted for ubiquitination by COP1 in the dark during skotomorphogenesis. With HDA15 being more stable than COP1, the direct interaction between HDA15 and COP1 leads us to conclude that HDA15 represses COP1.

\section{Discussion}

Histone active marks such as $\mathrm{H} 3 \mathrm{~K} 9 \mathrm{~K} 27 \mathrm{ac}$ signals transcriptional activation whereby light induces the acetylation of positive photomorphogenic regulators functioning as chlorophyll a/b-binding protein, peroxidase, citrate synthase, RBCS small chain 1B, ribosomal protein, PSIl-light-harvesting complex, $\mathrm{HYH}$, HY5 and its downstream targets CHS, IAA3, RBCS-1a during photomorphogenesis (Benhamed et al., 2006; Guo et al., 2008; Charron et al., 2009). On the other hand, histone deacetylation stimulates chromatin compaction triggering transcriptional quiescence. Among the RPD3/HDA1-like superfamily of HDAs, Class I histone deacetylases HDA6 and HDA19 display negative regulatory roles in photomorphogenesis. Together with PHYB, Tessadori et al. have identified HDA6 as a regulator of lightinduced chromatin condensation (Tessadori et al., 2009). Similarly, HDA19 is suspected of repressing many light-regulated genes by deacetylating $\mathrm{H} 3 \mathrm{~K} 9 \mathrm{~K} 14$ at the transcriptional start site of the phy A locus (Benhamed et al., 2006; Jang et al., 2011).

On the other hand, it appears that Class II HDAs play a positive regulatory role in the light signaling network with HDA15 repressing COP1 at its locus and modulating its activities via subcompartmentalization and direct interaction. As HDA5 and HDA18 also exhibit long-hypocotyl phenotypes at varying light treatments, it remains a conundrum of how these and other Class II HDAs function during photomorphogenesis. These findings fine-tune our prior understanding of the role of histone modifications in light-regulated gene expression. Light not only induces the acetylation of key positive regulators of photomorphogenesis but also renders the simultaneous repression of vital negative regulators such as COP1 via direct interaction. Taken together, our results indicated that histone acetyltransferases and deacetylases act as multi-functional and potent chromatin-modifying enzymes during photomorphogenesis.

While other mutant Class II HDAs also manifest light hyposensitivity, only HDA15 is driven by light to shuttle in and out of the nucleus. The environmental cue or stress that would signal the nuclear import of 
the other Class II HDAs and their functional characterization remain to be investigated as most of them have been observed to be cytoplasmic (Alinsug et al., 2012; Tran et al., 2012).

Histone deacetylases constitute a family of nuclear enzymes known primarily for their role in transcriptional repression. Class II HDAs, in particular, are known to be versatile regulators catalyzing the deacetylation of both histones and non-histone proteins. The mammalian HDAC6 modulates a wide variety of cellular activities involving complex interactions with a-tubulin, cortactin, HSP90, and other proteins (Matthias et al., 2008; Valenzuela-Fernandez et al., 2008). Similarly, HDAC1 complexes with MDM2, an E3 ubiquitin ligase, deacetylate p53, and promote its ubiquitination (Ito et al., 2002). In the case of AtHDA15, it attenuates COP1 modulating its repressive activities via direct interaction, subcellular compartmentalization, and chromatin compaction at its transcriptional start site. Based on our results, this versatile regulator not only functions as a traditional histone deacetylase silencing key regulators of photomorphogenesis, specifically COP1, but it also directly interacts with COP1 likely attenuating its E3 ligase activities.

The specific molecular mechanisms for the degradation and/or cytoplasmic export of COP1 in the nucleus under light conditions remain obscure. The nuclear interaction of HDA15 with COP1 under light conditions may support the notion that COP1's depletion may be enhanced by the binding of HDA15 to induce auto-ubiquitination. Based on studies conducted by Alinsug et al. (2020), overexpression of HDA15 significantly reduced COP1 protein levels and elevated HY5 protein concentrations in various light treatments. However, hda 15 mutant lines did not incur any changes in its COP1 protein levels. Thus, biochemical studies elucidating on the possibility of induced auto-ubiquitination of COP1 via HDA15 binding would be an interesting research path to pursue.

In the presence of light, COP1 is mainly cytoplasmic, although some trace amounts are left inside the nucleus. This purges HDA15 to shuttle into the nucleus binding to COP1 to attenuate its E3 ligase activity against HY5 regulating photomorphogenesis. In the dark, contrarily, COP1 exclusively localizes inside the nucleus and targets the ubiquitination of HY5 to advance skotomorphogenesis. While the majority of HDA15 translocates into the cytoplasm, partial amounts left in the nucleus target the deacetylation of the transcriptional start site of COP1 locus and/or interact with COP1 to modulate its repressive activities. The molecular switches in apportioning the exact amounts of HDA15 to remain active inside the nucleus while others are deactivated inducing their nuclear export remains elusive and requires further investigation.

Presented in Figure 8 is a working model depicting the sub-compartmentalization and modularized activities of COP1 and HDA15 under light and dark conditions. As light signals the nucleocytoplasmic shuttling of HDA15, its nuclear import is primarily dependent on the presence of COP1. Based on our findings, transient expression of HDA15-GFP using cop 1-4 mutant protoplasts exhibits cytoplasmic localization whether it is treated under white light or dark. Since HDA15 have contrasting localization with COP1 in the presence and absence of light, we hypothesize that a concentration gradient on either of the proteins influences the movement of the other in opposing directions, assuming these enzymes are not 
degraded; or, if a dominant ligand induces the degradation of the other. Thus, further studies are required to unravel the molecular underpinnings of the opposing nucleocytoplasmic translocation of HDA15 \& COP1 upon light and dark signaling cues.

We also attempted to determine the binding sites of the two repressors by generating different truncated constructs of COP1 and HDA15 containing various combinations of potential protein-binding domains. Surprisingly, only the full-length HDA15 was able to bind the full-length COP1 suggesting strict, proper protein conformation for such interaction.

Previous studies on the conserved domains of RPD3/HDA1-like superfamily show that Zn finger domains are imprinted on several Class II HDAs (Pandey et al., 2002; Alinsug et al., 2009). The monocot ortholog of AtHDA15 in maize, ZmHDA110, has a C3HC4 type RING Zn finger that binds with ubiquitin while the ZnFUBP of the mammalian Class II HDAC6 binds to ubiquitin specifically at the $\mathrm{C}$ termini diglycine motif catalyzed by a deubiquitinase, ataxin-3 (Ouyang et al., 2012). RanBP-ZnF in HDA15 may similarly interact with ubiquitin, such as those found in Npl4 (Alam et al., 2004). Based on sequence analyses on Zn fingers conducted by Wang et al., the molecules have highly conserved cysteine residues and other key motifs in binding ubiquitin (Wang et al., 2003). As illustrated in Figure 9, sequence comparison between HDA15's RanBP2-ZnF to the human Np14 (NZF) reveals the presence of the two canonical highlyconserved cysteine residues and a key ubiquitin-binding motif, $\mathrm{Thr}_{95}$. Considering the RING-ZnF domain of COP1 catalyzes the transfer of ubiquitin units from E2 transferases to target proteins such as HY5, we speculate that ubiquitin may constitute a recognition site for HDA15 to identify COP1 as an active E3 ligase.

Nevertheless, unlike HDAC6, which binds and transports ubiquitinated proteins to aggresomes, the binding of HDA15 to COP1 undertakes a regulatory role via the attenuation of the latter's E3 ligase activity. Based on bioinformatics analysis comparing the ubiquitination sites of HY5, COP1, and HDA15, it is predicted that HDA15 is more stable than COP1. As expected, HY5 is the least stable making it more prone to ubiquitination. Still, questions remain on the exact nature of binding of HDA15 to COP1 as well as the properties of the molecular switches downstream of the HDA15-COP1 complex.

\section{Declarations}

\section{Acknowledgments}

The authors are grateful to the unwavering support of the various laboratory professors, mentors, colleagues, and staff for the conduct of this research. Special mention goes to Prof. Keqiang Wu and Prof. Hsu-Liang Hsieh of NTU and Prof. Jeong Joo Cheong of SNU for their guidance and access to their facilities. MVA is equally indebted to the scholarship and research fellowship grants funded by the Democratic Pacific Union, National Science Council of Taiwan, National Taiwan University, and Seoul National University.

\section{Funding}


This work was supported by the Democratic Pacific Union PhD studentship and research fellowship grants from the National Science Council of Taiwan, National Taiwan University, and Seoul National University to MVA.

\section{Conflict of Interest}

The authors have no conflicts of interest to declare that are relevant to the content of this article.

\section{Data Availability}

The data supporting the findings of this study are available in the paper and from the corresponding author, Malona V. Alinsug, upon request.

\section{Code Availability}

NA

\section{Authors' Contribution}

All authors contributed to the study conception and design. Material preparation, data collection and analysis were performed by Malona V. Alinsug and Custer C. Deocaris. The first draft of the manuscript was written by Malona V. Alinsug and Custer C. Deocaris commented on previous versions of the manuscript. Both authors read and approved the final manuscript.

\section{Ethics Approval}

NA

\section{Consent to Participate}

NA

\section{Consent to Publish}

All authors read and approved the final manuscript.

\section{References}

1. Akimov V, Barrio-Hernandez I, Hansen SV, Hallenborg P, Pedersen, AK, Bekker-Jensen DB, Puglia M, Christensen SD, Vanselow JT, and Nielsen MM (2018) UbiSite approach for comprehensive mapping of lysine and N-terminal ubiquitination sites. Nat Struct \& Mol Biol 25:631-640

2. Alam SL, Sun J, Payne M, Welch BD, Blake BK, Davis DR, Meyer HH, Emr SD, \& Sundquist WI (2004) Ubiquitin interactions of NZF zinc fingers. EMBO J 23:1411-1421 
3. Alinsug MV, Yu CW, \& Wu K (2009) Phylogenetic analysis, subcellular localization, and expression patterns of RPD3/HDA1 family histone deacetylases in plants. BMC Plant Biol 9:37. https://doi.org/10.1186/1471-2229-9-37

4. Alinsug MV, Chen FF, Luo M, Tai R, Jiang L, \& Wu K (2012) Subcellular localization of class II HDAs in Arabidopsis thaliana: nucleocytoplasmic shuttling of HDA15 is driven by light. PLoS One 7:e30846. https://doi.org/10.1371/journal.pone.0030846

5. Alinsug MV, Radziejwoski A, \& Deocaris CC (2020) AtHDA15 binds directly to COP1 positively regulating photomorphogenesis. Biochem Biophysical Res Comm 533:806-812. https://org/10.1016/j.bbrc.2020.09.089

6. Ausin I, Alonso-Blanco C, \& Martinez-Zapater JM (2004) Environmental regulation of flowering. Int J of Dev Biol 49:689-705

7. Benhamed M, Bertrand C, Servet C, \& Zhou DX (2006) Arabidopsis GCN5, HD1, and TAF1/HAF2 interact to regulate histone acetylation required for light-responsive gene expression. Plant Cell 18:2893-2903

8. Benhamed M, Martin-Magniette ML, Taconnat L, Bitton F, Servet C, De Clercq R, De Meyer B, Buysschaert C, Rombauts S, Villarroel R, Aubourg S, Beynon J, Bhalerao RP, Coupland G, Gruissem W, Menke FL, Weisshaar B, Renou JP, Zhou DX, \& Hilson P (2008) Genome-scale Arabidopsis promoter array identifies targets of the histone acetyltransferase GCN5. Plant J Cell \& Mol Biol 56:493-504

9. Charron JB, He H, Elling AA, \& Deng XW (2009) Dynamic landscapes of four histone modifications during deetiolation in Arabidopsis. Plant Cell 21:3732-3748

10. Gendrel AV, Lippman Z, Martienssen R, \& Colot V (2005) Profiling histone modification patterns in plants using genomic tiling microarrays. Nature Methods 2:213-218

11. Gu D, Chen CY, Zhao M, Zhao L, Duan X, Duan J, Wu K, and Liu X (2017) Identification of HDA15-PIF1 as a key repression module directing the transcriptional network of seed germination in the dark. Nucleic Acids Res 45:7137-7150

12. Guo L, Zhou J, Elling AA, Charron JB, \& Deng, XW (2008) Histone modifications and expression of light-regulated genes in Arabidopsis are cooperatively influenced by changing light conditions. Plant Physiol 147:2070-2083

13. Guruprasad K, Reddy BB, \& Pandit MW (1990) Correlation between stability of a protein and its dipeptide composition: a novel approach for predicting in vivo stability of a protein from its primary sequence. Protein Eng Design \& Sel 4:155-161

14. Holm M \& Deng XW (1999) Structural organization and interactions of COP1, a light-regulated developmental switch. Plant Mol Biol 41:151-158

15. Hook SS, Orian A, Cowley SM, \& Eisenman RN (2002) Histone deacetylase 6 binds polyubiquitin through its zinc finger (PAZ domain) and copurifies with deubiquitinating enzymes. Proc Nat Acad Sci of the USA 99:13425-13430

16. Hsieh HL, Okamoto H, Wang M, Ang LH, Matsui M, Goodman H, \& Deng XW (2000) FIN219, an auxinregulated gene, defines a link between phytochrome $A$ and the downstream regulator COP1 in light 
control of Arabidopsis development. Genes \& Dev 14: 1958-1970

17. Ito A, Kawaguchi Y, Lai CH, Kovacs JJ, Higashimoto Y, Appella E, \& Yao TP (2002) MDM2-HDAC1mediated deacetylation of p53 is required for its degradation. EMBO 21:6236-6245

18. Jang IC, Chung PJ, Hemmes H, Jung C, \& Chua NH (2011) Rapid and reversible light-mediated chromatin modifications of Arabidopsis phytochrome A locus. Plant Cell 23:459-470

19. Jiao Y, Lau OS, \& Deng XW (2007) Light-regulated transcriptional networks in higher plants. Nature reviews. Genetics 8:217-230

20. Kovacs JJ, Cohen TJ, \& Yao TP (2005) Chaperoning steroid hormone signaling via reversible acetylation. Nucl Rec Sig 3:e004.

21. Kumar D \& Kumar P (2019) An In-Silico Investigation of Key Lysine Residues and Their Selection for Clearing off $A \beta$ and Holo-AßPP Through Ubiquitination. Interdisciplinary Sciences. Comput Life Sci 11:584-596.

22. Kurdistani SK, Tavazoie S, \& Grunstein M (2004) Mapping global histone acetylation patterns to gene expression. Cell 117:721-733

23. Li B, Krishnan VG, Mort ME, Xin F, Kamati KK, Cooper DN, Mooney SD, \& Radivojac P (2009) Automated inference of molecular mechanisms of disease from amino acid substitutions. Bioinfo 25:2744-2750.

24. Li A, Xue Y, Jin C, Wang M, \& Yao X (2006) Prediction of N-acetylation on internal lysines implemented in Bayesian Discriminant Method. Biochem Biophys Res Commun 350:818-24

25. Liu X, Wei W, Zhu W, Su L, Xiong Z, Zhou M, Zheng Y, \& Zhou DX (2017) Histone Deacetylase AtSRT1 Links Metabolic Flux and Stress Response in Arabidopsis. Mol Plant 10:1510-1522

26. Liu X, Chen CY, Wang KC, Luo M, Tai R, Yuan L, Zhao M, Yang S, Tian G, Cui Y, Hsieh HL, \& Wu K (2013) PHYTOCHROME INTERACTING FACTOR3 associates with the histone deacetylase HDA15 in repression of chlorophyll biosynthesis and photosynthesis in etiolated Arabidopsis seedlings. Plant Cell 25:1258-1273

27. Luxton GG \& Gundersen GG (2007) HDAC6-pack: cortactin acetylation joins the brew. Dev Cell 13:161-162

28. Ma X, Lv S, Zhang C, \& Yang C (2013) Histone deacetylases and their functions in plants. Plant Cell Reports 32:465-478

29. Matthias P, Yoshida M, \& Khochbin S (2008) HDAC6 a new cellular stress surveillance factor. Cell Cycle 7:7-10

30. Osterlund MT, Hardtke CS, Wei N, \& Deng XW (2000) Targeted destabilization of HY5 during lightregulated development of Arabidopsis. Nature 405:462-466

31. Ouyang, H., Ali, Y.O., Ravichandran, M., Dong, A., Qiu, W., MacKenzie, F., Dhe-Paganon, S., Arrowsmith CH \& Zhai RG (2012) Protein aggregates are recruited to aggresome by histone deacetylase 6 via unanchored ubiquitin C termini. J Biol Chem 287, 2317-2327 
32. Pandey R, Muller A, Napoli CA, Selinger DA, Pikaard CS, Richards EJ, Bender J, Mount DW, \& Jorgensen RA (2002) Analysis of histone acetyltransferase and histone deacetylase families of Arabidopsis thaliana suggests functional diversification of chromatin modification among multicellular eukaryotes. Nucleic Acids Res 30:5036-5055

33. Schenke D, Cai D, \& Scheel D (2014) Suppression of UV-B stress responses by flg22 is regulated at the chromatin level via histone modification. Plant, Cell \& Envi 37: 1716-1721

34. Schübeler D, MacAlpine DM, Scalzo D, Wirbelauer C, Kooperberg C, van Leeuwen F, Gottschling DE, O'Neill LP, Turner BM, \& Delrow J (2004) The histone modification pattern of active genes revealed through genome-wide chromatin analysis of a higher eukaryote. Genes \& Dev 18:1263-1271

35. Tang Y, Liu X, Liu X, Li Y, Wu K, \& Hou X (2017) Arabidopsis NF-YCs Mediate the Light-Controlled Hypocotyl Elongation via Modulating Histone Acetylation. Mol Plant 10:260-273

36. Tessadori F, van Zanten M, Pavlova P, Clifton R, Pontvianne F, Snoek LB, Millenaar FF, Schulkes RK, van Driel R, Voesenek LA, Spillane C, Pikaard CS, Fransz P, \& Peeters AJ (2009) Phytochrome B and histone deacetylase 6 control light-induced chromatin compaction in Arabidopsis thaliana. PLoS Genetics 5:e1000638

37. Tran HT, Nimick M, Uhrig RG, Templeton G, Morrice N, Gourlay R, DeLong A, \& Moorhead GB (2012) Arabidopsis thaliana histone deacetylase 14 (HDA14) is an alpha-tubulin deacetylase that associates with PP2A and enriches in the microtubule fraction with the putative histone acetyltransferase ELP3. Plant J : Cell \& Mol Biol 71:263-272

38. Valenzuela-Fernandez A, Cabrero JR, Serrador JM, \& Sanchez-Madrid F (2008) HDAC6: a key regulator of cytoskeleton, cell migration and cell-cell interactions. Trends in Cell Biol 18:291-297

39. Wang B, Alam SL, Meyer HH, Payne M, Stemmler TL, Davis DR, \& Sundquist WI (2003) Structure and ubiquitin interactions of the conserved zinc finger domain of Npl4. J Biol Chem 278:20225-20234

40. Yang JY, Iwasaki M, Machida C, Machida Y, Zhou X, \& Chua NH (2008) BetaC1, the pathogenicity factor of TYLCCNV, interacts with AS1 to alter leaf development and suppress selective jasmonic acid responses. Genes \& Dev 22:2564-2577

41. Yoo SD, Cho YH, \& Sheen J (2007) Arabidopsis mesophyll protoplasts: a versatile cell system for transient gene expression analysis. Nature Protocols 2:1565-1572

42. Zhao L, Peng T, Chen CY, Ji R, Gu D, Li T, Zhang D, Tu YT, Wu K, \& Liu X (2019) HY5 Interacts with the Histone Deacetylase HDA15 to Repress Hypocotyl Cell Elongation in Photomorphogenesis. Plant Physiol 180:1450-1466

43. Zhou R, Han L, Li G, \& Tong T (2009) Senescence delay and repression of p16INK4a by Lsh via recruitment of histone deacetylases in human diploid fibroblasts. Nucleic Acids Res 37:5183-5196

\section{Tables}


Table I. Predicted ubiquitinated residues in AtHDA15, COP1 and HY5 based on three algorithms.

\begin{tabular}{|c|c|c|c|c|c|c|c|}
\hline & ID & Length & Ub-Pred * & BDM-PUB ${ }^{* *}$ & UbiSite $^{* \pi *}$ & Consensus & $\begin{array}{l}\text { Instability } \\
\text { Index } x^{* * * *}\end{array}$ \\
\hline AtHDA 15 & Q8GXJ1 & 552 aа. & $\begin{array}{l}14(0.75) ; 129(0.65) ; 139 \\
(0.80) ; 167(0.69)\end{array}$ & $\begin{array}{l}14(2.34) ; 46(2.87) ; 113(0.31) ; \\
129(0.76) ; 139(0.59) ; 265 \\
(0.90) ; 305(1.25) ; 306(1.96) ; \\
375(1.26) ; 435(2.14) ; 458 \\
(1.49) ; 503(2.08) ; 516(0.48) ; \\
517(0.54) ; 521(0.88) ; 523 \\
(1.02) ; 552(4.49)\end{array}$ & $\begin{array}{l}46(0.55) ; 129(0.38) ; 139 \\
(0.45) ; 167(0.62) ; 205(0.40) ; \\
265(0.58) ; 305(0.67) ; 306 \\
(0.53) ; 328(0.46) ; 375(0.31) ; \\
458(0.35) ; 503(0.33) ; 532 \\
(0.25) ; 536(0.30) ; 537(0.35)\end{array}$ & $129 ; 139$ & $\begin{array}{l}37.11 \\
\text { (stable) }\end{array}$ \\
\hline COP1 & P43254 & 675 aа. & $\begin{array}{l}14(0.80) ; 193(0.60) ; 273 \\
(0.84) ; 274(0.96) ; 312(0.74) ; \\
328(0.76) ; 433(0.66)\end{array}$ & $\begin{array}{l}14(1.51) ; 106(2.66) ; 109 \\
(0.96) ; 110(1.17) ; 118(1.86) ; \\
139(1.35) ; 152(1.87) ; 154 \\
(0.70) ; 176(0.56) ; 178(1.40) ; \\
216(1.21) ; 263(2.02) ; 273 \\
(1.89) ; 274(2.79) ; 295(0.38) ; \\
296(0.92) ; 323(0.91) ; 328 \\
(1.76) 396(1.90) ; 512(1.04) ; \\
549(1.10) ; 550(0.73) ; 556 \\
(1.65) ; 653(0.73) ; 669(1.04)\end{array}$ & $\begin{array}{l}14(0.47) ; 106(0.47) ; 109 \\
(0.34) ; 110(0.28) ; 118(0.62) ; \\
152(0.43) ; 154(0.40) ; 176 \\
(0.39) ; 178(0.33) ; 193(0.23) ; \\
216(0.44) ; 234(0.29) ; 258 \\
(0.56) ; 259(0.47) ; 263(0.23) ; \\
273(0.50) ; 274(0.51) ; 312 \\
(0.26) ; 323(0.36) ; 328(0.28) ; \\
396(0.20) ; 422(0.21) ; 464 \\
(0.48) ; 512(0.30) ; 549(0.23) ; \\
550(0.45) ; 556(0.27) ; 578 \\
(0.50) ; 593(0.56) ; 619(0.28) ; \\
669(0.35)\end{array}$ & $\begin{array}{c}14 ; 193 ; 273 \\
274 ; 328\end{array}$ & $\begin{array}{c}47.13 \\
\text { (unstable) }\end{array}$ \\
\hline HY5 & $\mathrm{O} 24646$ & 168 aа. & $\begin{array}{l}31(0.95) ; 53(0.83) ; 79(0.68) ; \\
110(0.70) ; 121(0.80) ; 126 \\
(0.87)\end{array}$ & $\begin{array}{l}31(0.86) ; 53(1.95) ; 79(1.32) \\
87(3.02) ; 90(2.34) ; 93(1.64) \\
109(1.65) ; 110(1.05) ; 126 \\
(0.87) ; 155(1.58)\end{array}$ & $\begin{array}{l}31(0.38) ; 53(0.43) ; 79(0.24) ; \\
90(0.38) ; 109(0.33) ; 110 \\
(0.38) ; 121(0.42) ; 126(0.46) ; \\
149(0.28) ; 155(0.38)\end{array}$ & $\begin{array}{c}31 ; 53 ; 79 ; 110 ; \\
126\end{array}$ & $\begin{array}{c}68.21 \\
\text { (unstable) }\end{array}$ \\
\hline
\end{tabular}

* green (low confidence): score range $(0.62 \leq s \leq 0.69$ ), sensitivity $(0.464)$, specificity $(0.903)$; blue (medium confidence): score range $(0.69 \leq s \leq 0.84)$, sensitivity $(0.346)$, specificity $(0.950)$; red (high confidence): score rang e $(0.84 \leq s \leq 1.00)$, sensitivity $(0.197)$, specificity $(0.989)$

$* *$ Threshold $=0.3$

${ }^{* * *}$ Low threshold; green (low confidence level): $s c$ ore range $(s \leq 0.50)$, sensitivity $(0.8648)$, specificity $(0.3396)$; red (high confidence level): score range $(0.75 \leq s)$, sen sitivity $(0.3754)$, specificity $(0$. 8628)

${ }^{* * * *}$ predicted using Protparam tool, the instability index is an estimate of the stability of your protein in vitro

\section{Figures}




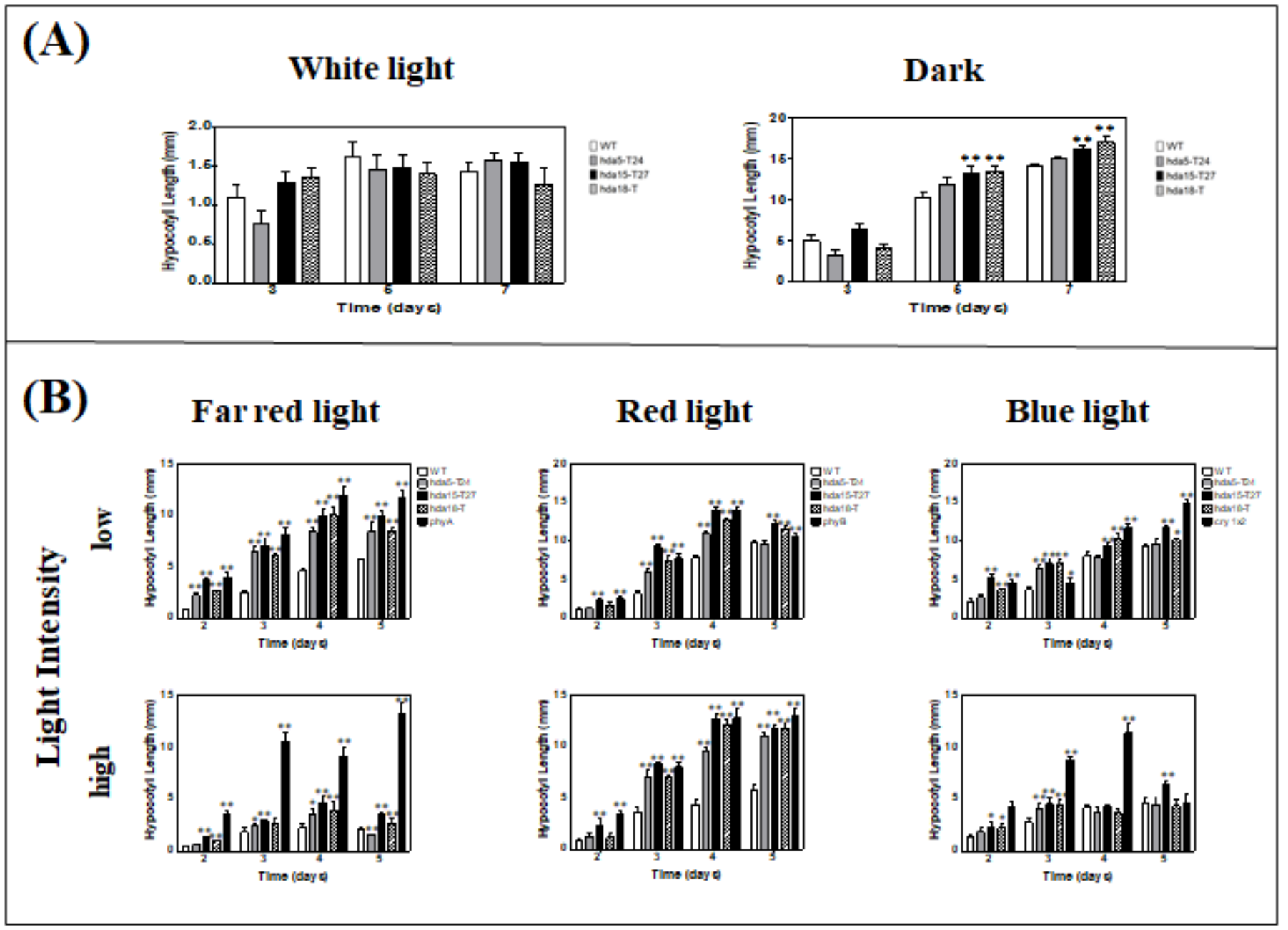

Figure 1

Comparison of light response of A. thaliana bearing class II HDA and phytochrome receptor mutations. (A) Photographs and hypocotyl lengths of WT, hda5, hda15, and hda18 mutants under to the dark and white light for seven consecutive days. (B) Hypocotyl lengths of the Class II HDA mutants concerning the phytochrome receptor mutants under far-red, red and blue light treatments at different light intensities for seven days. Light intensity treatments were designated as low $(\mathrm{L}$, upper panel) or high $(\mathrm{H}$, lower panel): far-red, $\mathrm{H}$ at 18.065 and $\mathrm{L}$ at $2.77 \mu \mathrm{mol} \mathrm{m}-2 \mathrm{~s}-1$; red light, $\mathrm{H}$ at 22.938 and $\mathrm{L}$ at $1.77 \mu \mathrm{mol} \mathrm{m}-2 \mathrm{~s}-1$; and blue light, $\mathrm{H}$ at 11.095 and $\mathrm{L}$ at $3.84 \mu \mathrm{mol} \mathrm{m}-2 \mathrm{~s}-1$. Data presented are mean values with error bars set from its standard deviation. Asterisks indicate significant difference, where single asterisk at $95 \%$ and double asterisk at $99 \%$ level of confidence 
White

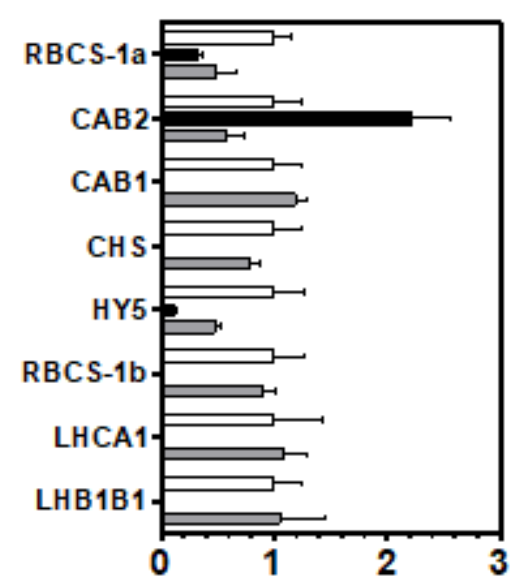

Normalized Fold Expression
Far Red

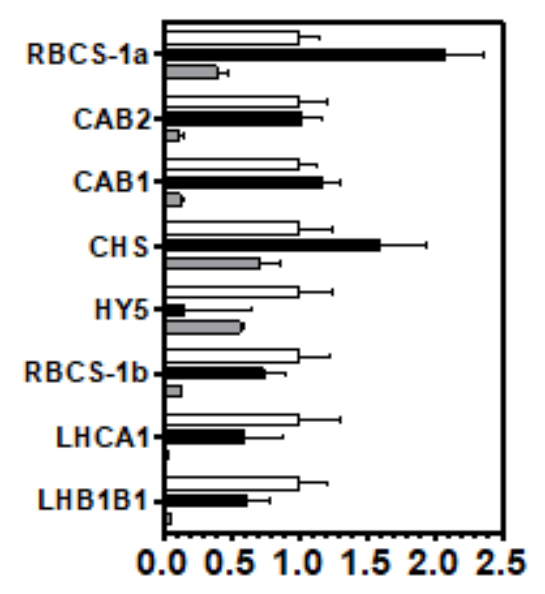

Normalized Fold Expression

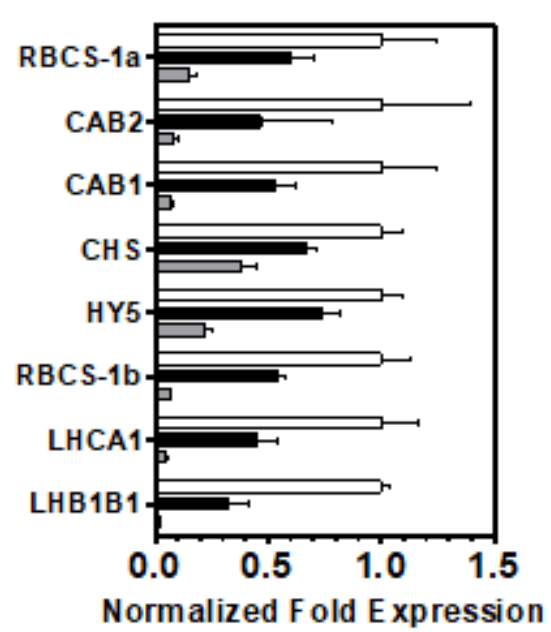

Normalized Fold Expression

$\square$ WT $\square$ hda15-t27 $\square$ phyA

\section{Figure 2}

Down-regulation of positive regulators to photomorphogenesis in AtHDA15 knockout lines. mRNA expression levels of positive regulators of photomorphogenesis were assessed in A. thaliana exposed to (A) white light, (B) far-red light, and (C) the dark. Expression levels reflected were normalized to ubiquitin 


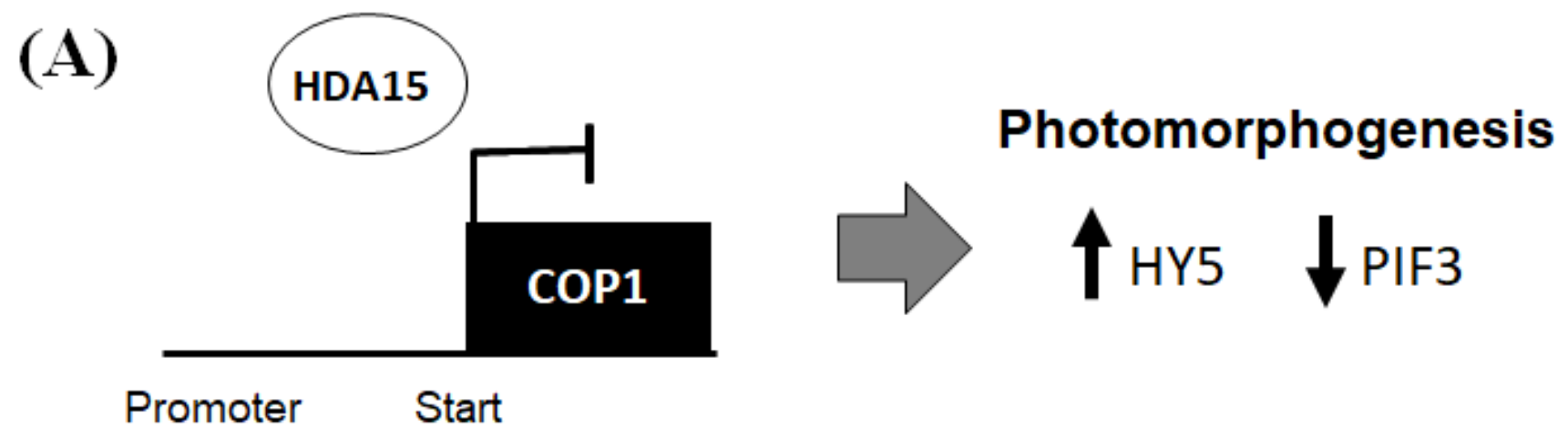

(B)

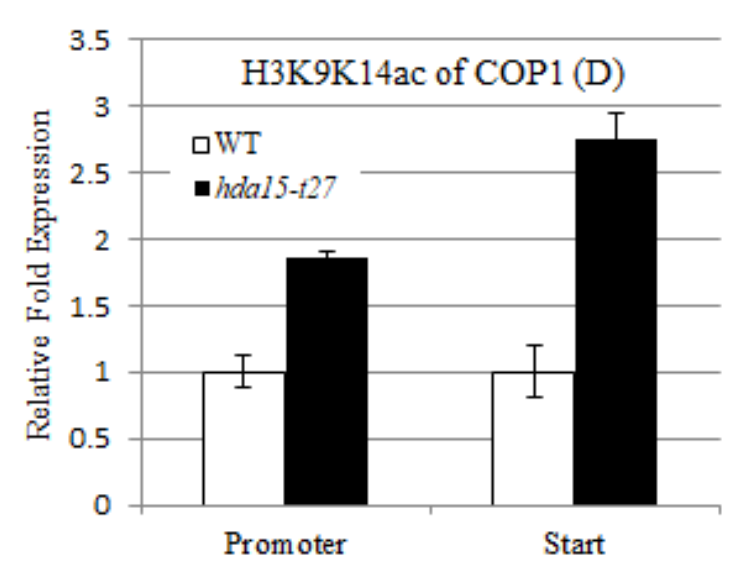

(C)

White

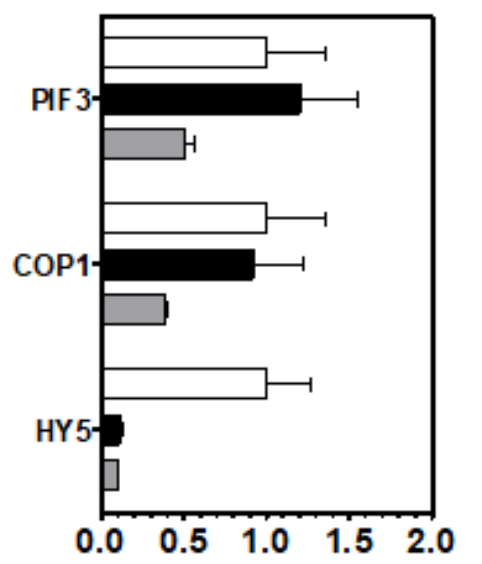

Normalized Fold Expression
Far Red

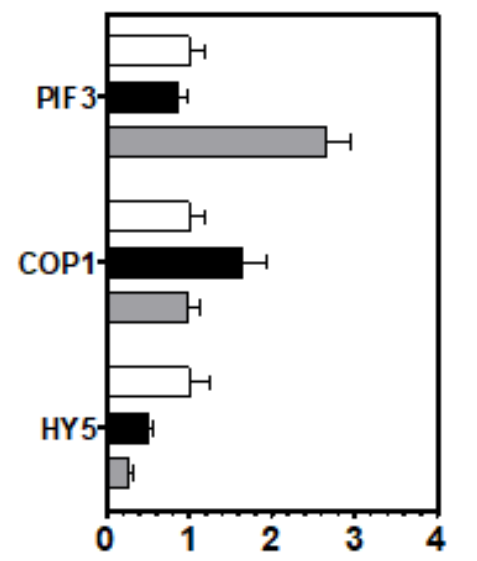

Normalized Fold Expression
Dark

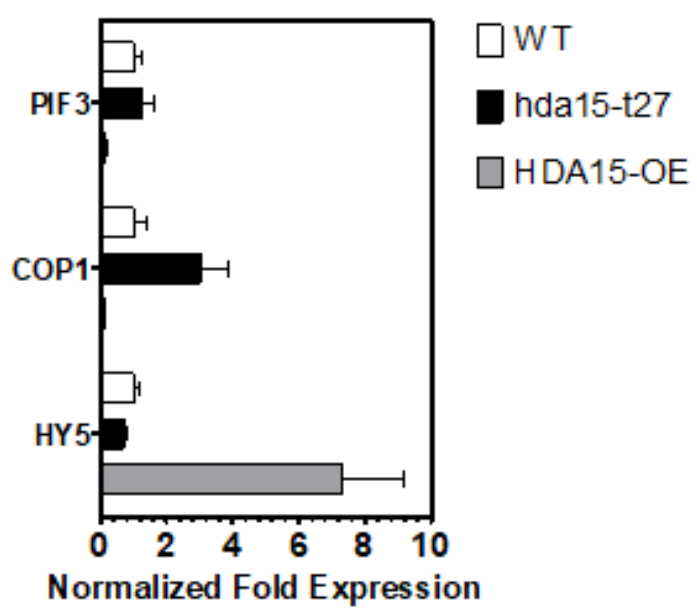

\section{Figure 3}

mRNA expression profiles of key regulators of photomorphogenesis in HDA15 mutant and overexpression lines. (A) Schematic diagram of repressive action of HDA15 on COP1 locus. (B) ChIP assay using anti-H3K9K14ac further reveals that the promoter \& start sites of COP1 were highly acetylated in the absence of HDA15 in dark-treated plants. (C) Compared to white light and far red light treated plants, dark-treatment has eminently amplified HY5 transcripts in HDA15 overexpression lines. 
Concomitantly, mRNA levels of COP1 were significantly elevated in hda15 mutants while its overexpression lead to COP1's extreme downregulation

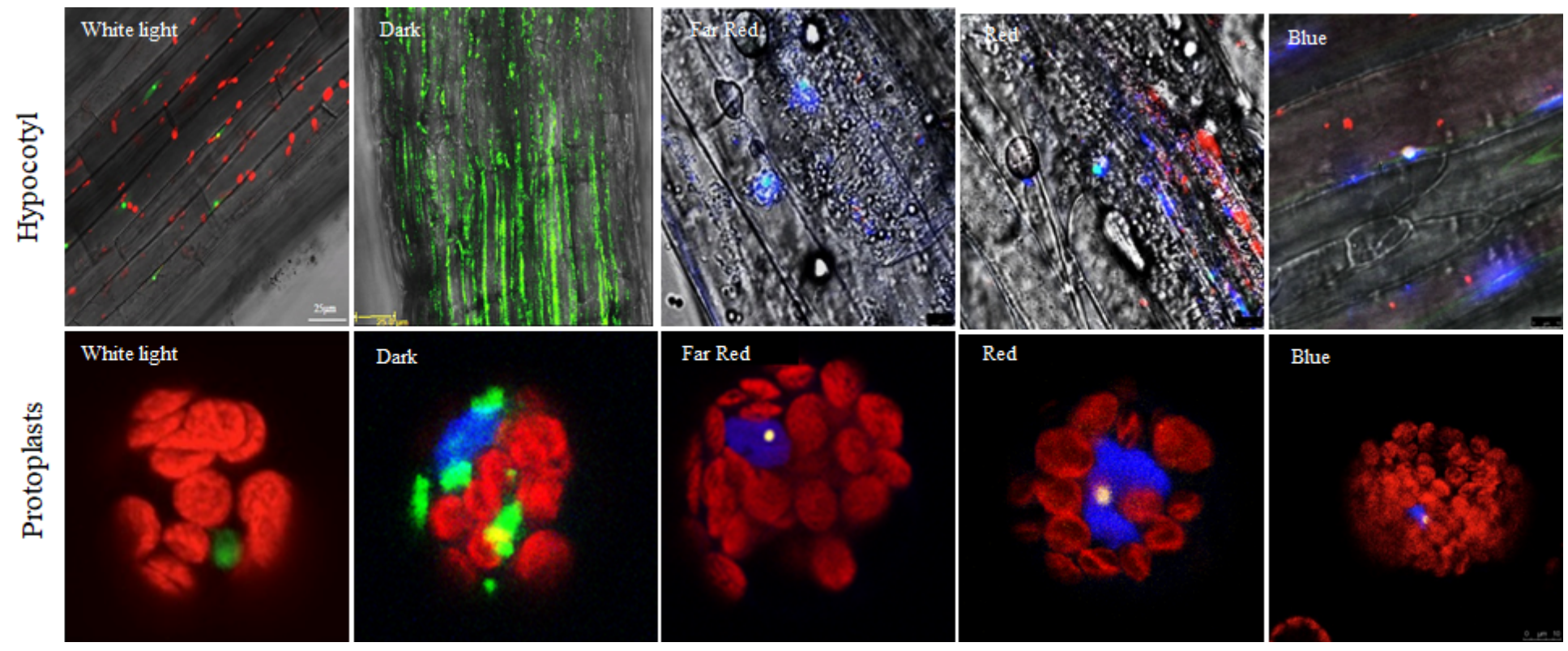

\section{Figure 4}

Subcellular localization of AtHDA15 under different light treatments. HDA15-GFP/YFP was localized in the nuclei of hypocotyls and protoplasts of transgenic lines grown under white light and different light treatments. Note: In dark treated plants, HDA15 has a pan-cytoplasmic distribution. Hoechst was used as nuclear marker

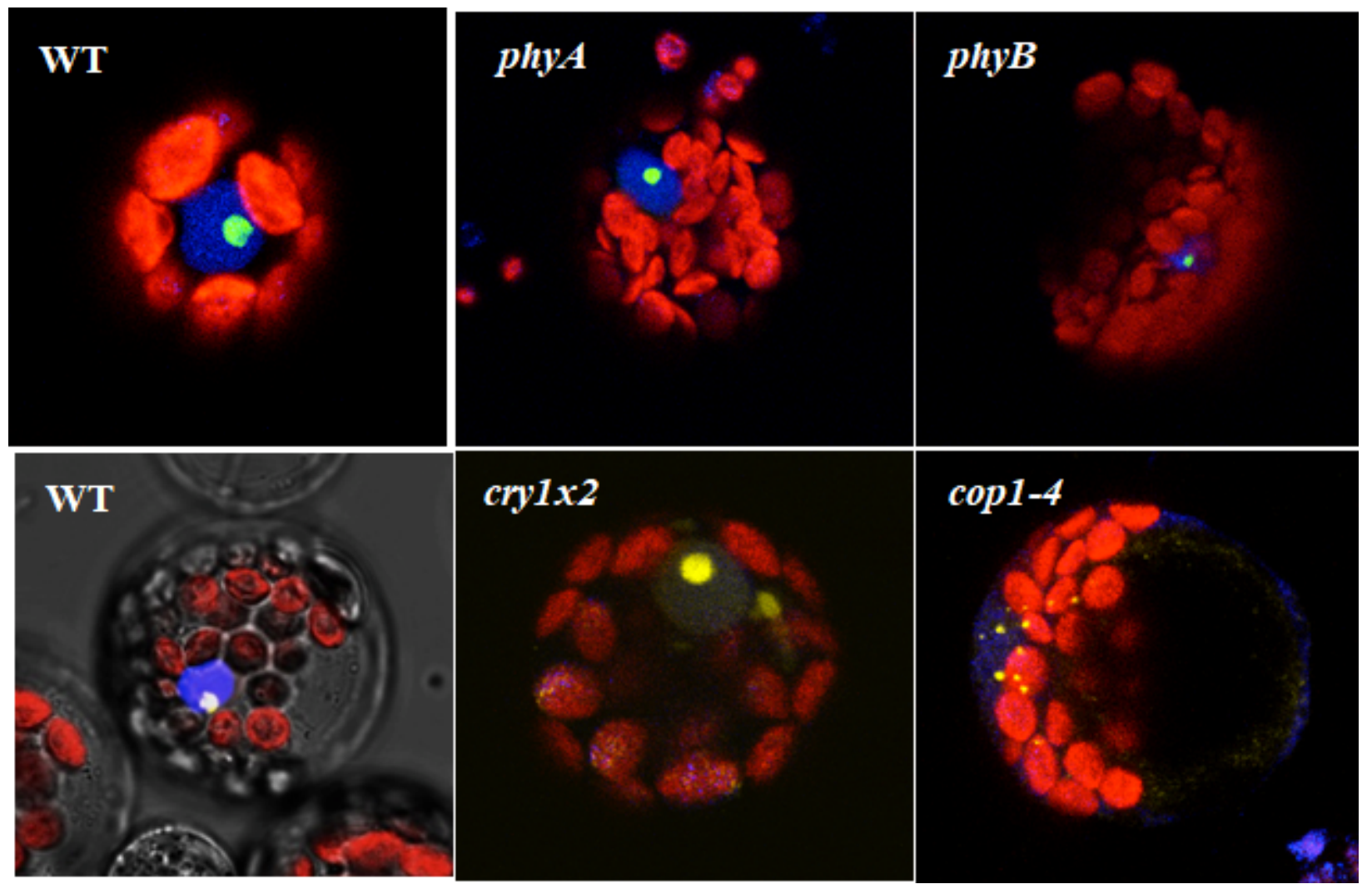

Figure 5 
COP1 activates nuclear localization of HDA15. HDA15 localization in protoplasts of mutant photoreceptors were all nuclear. However, when transiently expressed in cop1-4 mutant protoplasts, HDA15 exhibited cytoplasmic localization indicating that COP1 ultimately influences nuclear localization of HDA15. VirD2-NLS was co-transfected as nuclear marker (blue)

\section{(A)}

$\mathrm{kDa}$
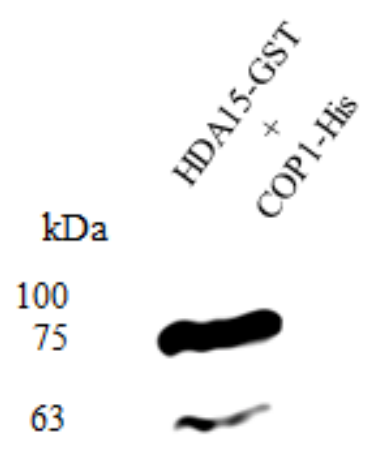

48

35

28
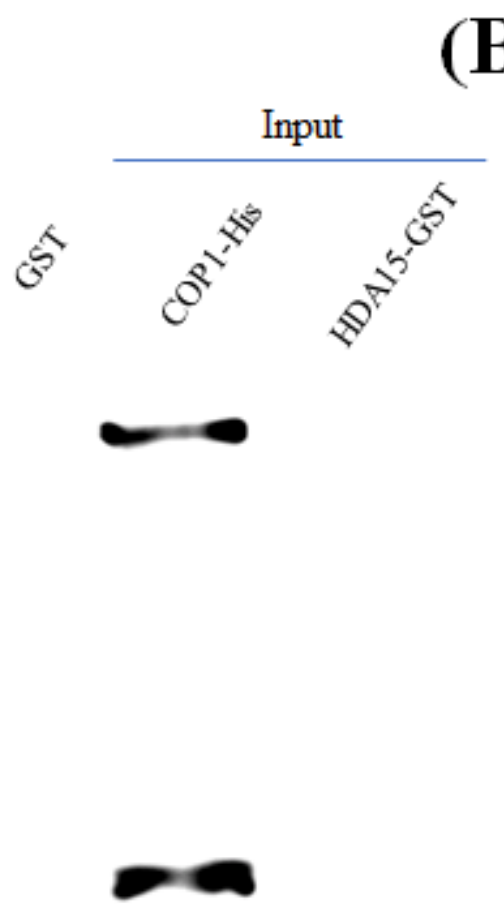

\section{(B)}
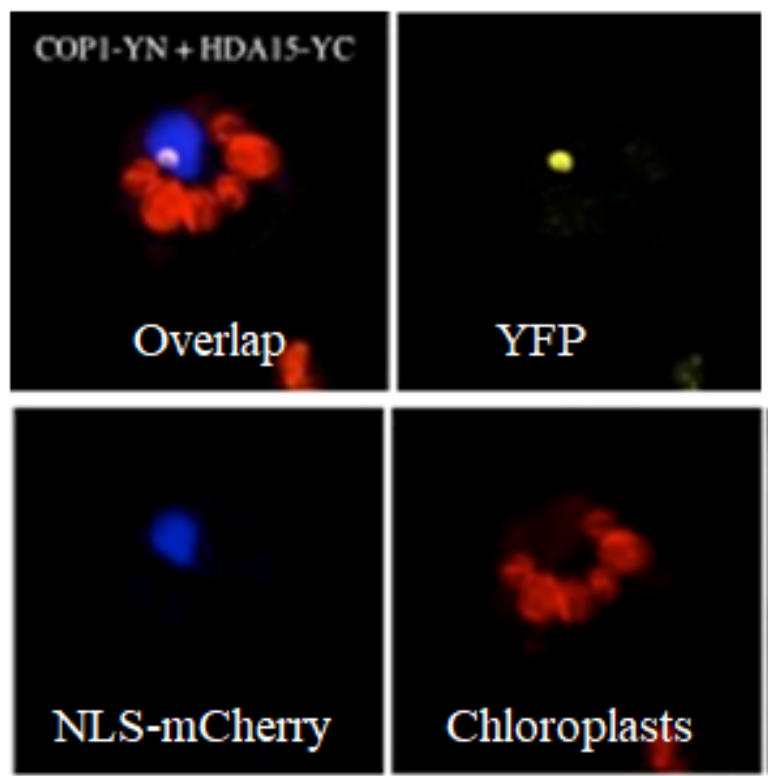

\section{Figure 6}

AtHDA15 directly associates with COP1. (A) In vitro and (B) in vivo interaction between COP1 and AtHDA15 indicates their direct binding inside the nucleus. AtHDA15-COP1 interaction was further confirmed via pull down assay using anti-His antibody where HDA15 was fused with GST and COP1 with His tags. Interaction of COP1 and AtHDA15 in isolated protoplasts was visualized 18-24 hr after transfection using bifluorescence complementation assay under laser confocal microscopy. AtHDA15 and COP1 genes were recombined with N-YFP and C-YFP. VirD2-NLS was used as nuclear marker 


\begin{tabular}{|c|c|c|}
\hline COP1 & HDA15 & Phenotype \\
\hline+ & + & Normal \\
+ & - & Long hypocotyl \\
\hline- & + & Short hypocotyl, Dwarfed \\
\hline- & - & Short hypocotyl, Dwarfed \\
\hline
\end{tabular}

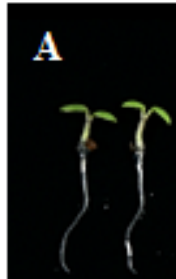

$\mathrm{Col}$

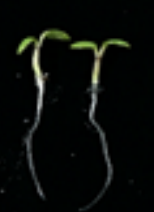

hdal $5-127$

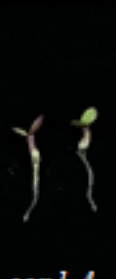

copl-4

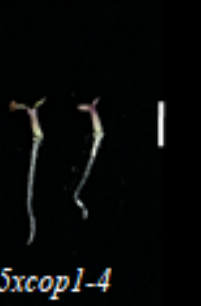

$15 x \operatorname{cop} 1-4$
B

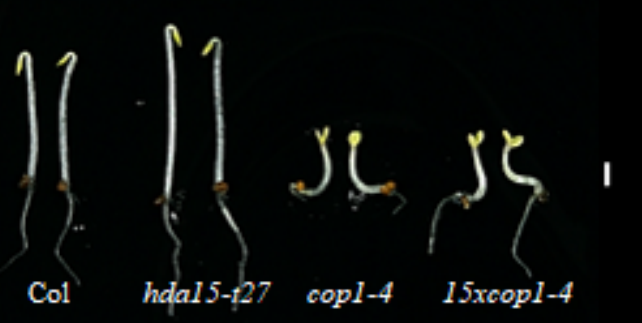

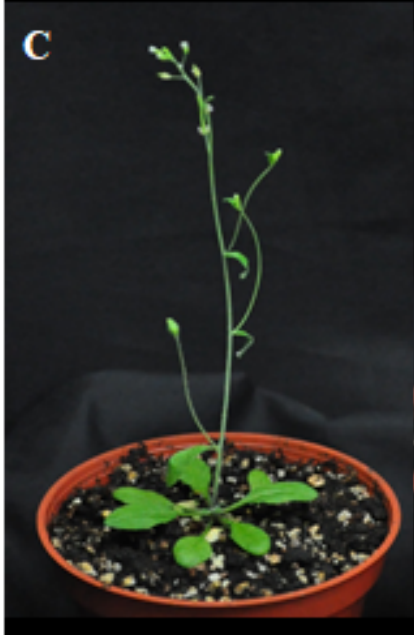

WT

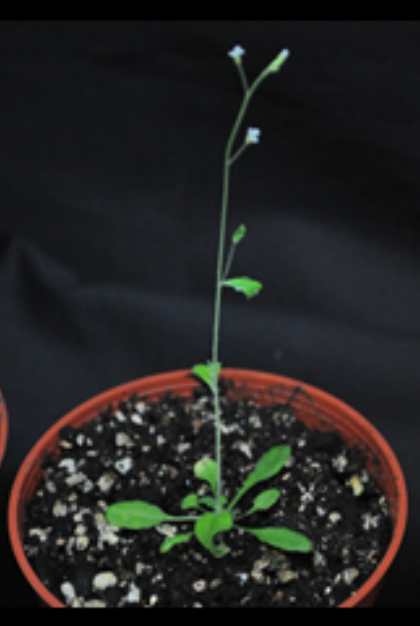

hdal5-t27

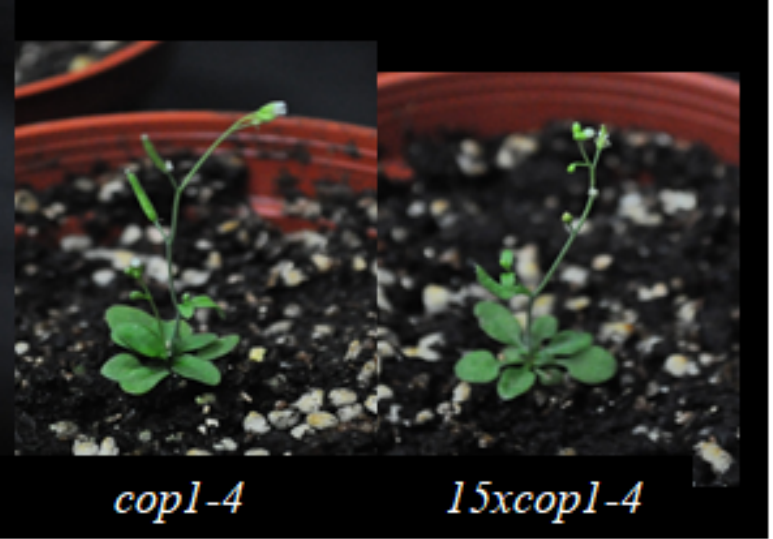

Figure 7

Double mutant hda15-t27xcop1-4 phenotype. The cross between hda15-t27 and cop1-4 mutants exhibited short hypocotyl phenotypes under white light $(A)$ and dark $(B)$ treatment for four days and dwarfed phenotypes in 3-week old plants (C) which are reminiscent of cop1 mutants. This indicates that COP1 is epistatic to HDA15 

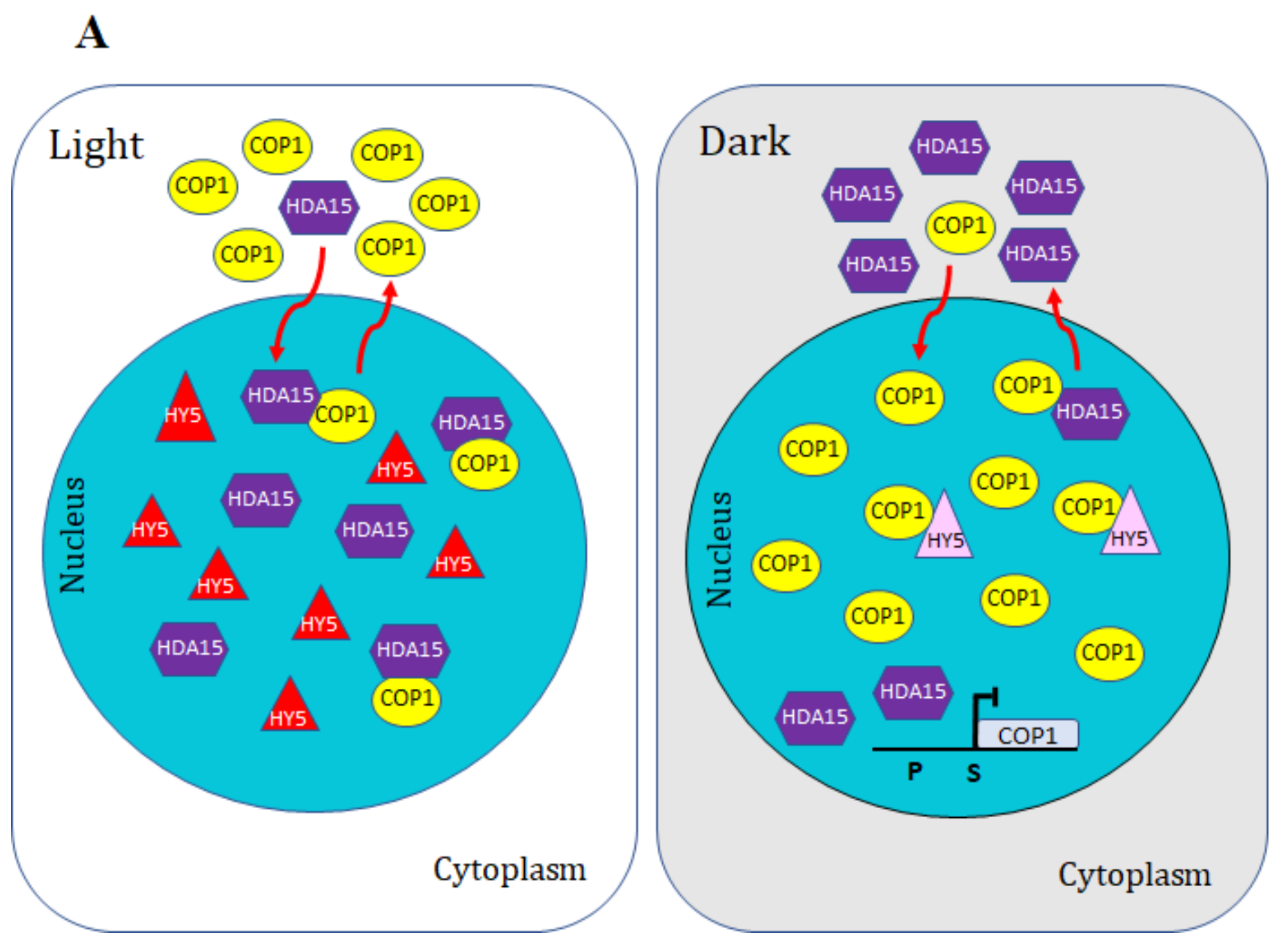

Figure 8

Working hypothesis illustrating modular activities and sub-compartmentalization of COP1 and HDA15 under light and dark conditions. The nucleocytoplasmic movement of HDA15 follows a concentration gradient of COP1 in opposing directions. In the presence of light, COP1 translocates into the cytoplasm purging HDA15 to be nuclear. With some amounts left inside the nucleus, HDA15 binds to COP1 attenuating its E3 ligase activity. In the dark, however, COP1 exclusively localizes inside the nucleus targeting the ubiquitination of HY 5 proceeding to skotomorphogenesis. This forces the majority of HDA15 to shuttle into the cytoplasm with some amounts left in the nucleus targeting deacetylation of COP1 start site for transcriptional repression

NZF

RanBP2

HDA15-RanBP2

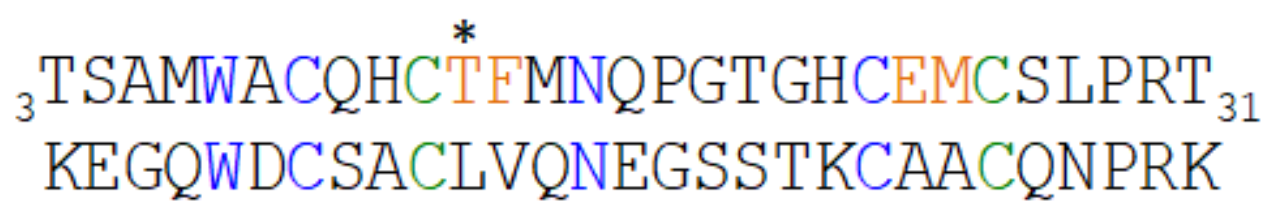
${ }_{86}$ FVKWCCVNCTMSNPGDMVHCCICGEHKES $_{115}$

Figure 9 
Sequence comparison between Zn fingers in human Npl4 (NZF), wild type RanBP2, which has minimal Ubq binding, and RanBP2 in AtHDA15. Conserved residues in NZF were defined by Wang et al. (2003), where blue letters indicate semi-conserved residues, green cysteine residues are highly conserved and contact Ubq, and orange residues as key motifs in binding Ubq 